Z Herz- Thorax- Gefäßchir 2020 · 34:62-68 https://doi.org/10.1007/s00398-019-00350-5 Online publiziert: 17. Dezember 2019 ○ Der/die Autor(en) 2019

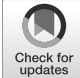

\section{Julia Riebandt ${ }^{1}$. Jasmin S. Hanke ${ }^{2}$}

${ }^{1}$ Klinische Abteilung für Herzchirurgie, Universitätsklinik für Chirurgie, Medizinische Universität Wien, Wien, Österreich

${ }^{2}$ Abteilung für Herz-, Thorax-, Transplantations- und Gefäßchirurgie, Medizinische Hochschule Hannover, Hannover, Deutschland

\title{
Der Herzinsuffizienzchirurg - Hausarzt und Herzchirurg
}

\section{„Epidemie“ Herzinsuffizienz}

Im Jahr 2015 waren Herz-KreislaufErkrankungen mit $39 \%$ die häufigste Todesursache in Deutschland [1]. Zudem steigt die Zahl der Erkrankten stetig [2]. Jährlich behandeln Kliniken über 1,7 Mio. vollstationäre Patienten aufgrund von Herzerkrankungen wie beispielsweise der koronaren Herzkrankheit, Herzklappenerkrankungen oder auch Herzinsuffizienz [3]. Die Herzinsuffizienz belegte mit 47.414 Todesfällen 2015 Platz 3 der Liste der 10 häufigsten Todesursachen [1]. Weiterhin leiden ca. $0,5-1 \%$ der deutschen Gesamtbevölkerung an einer Herzinsuffizienz, was in etwa 80.000 Neuerkrankungen/ Jahr entspricht [1-3]. Die Prävalenz der Herzinsuffizienz beträgt weltweit 1-2\%, steigt jedoch mit höherem Alter auf bis zu 22\% an [4-6]. Die „All-cause“-Einjahresmortalität beträgt $23 \%$ bei akuter Herzinsuffizienz und $7 \%$ bei chronischer „ambulanter“ Herzinsuffizienz. Die Rehospitalisationsraten sind sehr hoch (bis zu 43\%). Damit handelt sich bei der Herzinsuffizienz durchaus um ein sozioökonomisch relevantes Krankheitsbild $[7,8]$.

\section{》) Die Herzinsuffizienz belegte 2015 Platz 3 der Liste der 10 häufigsten Todesursachen}

Herzinsuffizienz ist ein multifaktorielles Krankheitsbild und ist definiert als klinisches Syndrom mit charakteristischen Symptomen und klinischer Manifestation. Herzinsuffizienz wird primär über die linksventrikuläre Auswurffraktion (LVEF) definiert. Differenzierungen können anhand folgender Faktoren vorgenommen werden [9]:

- erhaltene, mäßig eingeschränkte oder reduzierte LVEF:

- „heart failure with preserved ejection fraction" (HFpEF) EF $\geq 50 \%$,

- „heart failure with mid-range ejection fraction" (HFmEF). EF: 40-49\%,

- „heart failure with reduced ejection fraction" (HFrEF): EF < $40 \%$,

- zeitlicher Verlauf:

- akut vs. chronisch,

- stabil vs. dekompensiert und

- Schweregrad der Symptome.

Guidelines der European Association of Cardiology (ESC) geben für diese Patienten einen Therapieplan, bestehend aus Angiotensinkonversionsenzym(ACE)-

Hemmern und $\beta$-Rezeptoren-Blockern, als First-line-Therapie vor, die im zweiten Schritt um einen Mineralokortikoidrezeptorantagonisten und den Ersatz des ACE-Hemmers durch einen Angiotensinrezeptor-Neprilysin-Hemmer erweitert werden können. Im dritten Schritt wird die Evaluierung für eine kardiale Resynchronisationstherapie (CRT) vorgesehen. Stauungssymptome können durch Diuretikagabe gemildert werden [9].

Patienten, die trotz optimaler medikamentöser Therapie (OMT) symptomatisch bleiben, erreichen das Stadium der chronischen terminalen Herzinsuffizienz und sind für weiterführende interventio- nelle oder chirurgische Therapiekonzepte $\mathrm{zu}$ evaluieren.

\section{Chirurgische Therapieoptionen}

Abhängig von Ursache und Schweregrad der Herzinsuffizienz stehen neben medikamentösen auch verschiedene chirurgische Therapieoptionen zur Verfügung. Zugrunde liegende pathologische Veränderungen sollten also stets sorgfältig evaluiert werden, da sich unterschiedliche Therapiemöglichkeiten und Präventionsmöglichkeiten eines Voranschreitens der Erkrankung bzw. der Verhinderung des Versterbens des Patienten ergeben können.

\section{Antiarrhythmische Devices}

\section{„Implantable cardioverter- defibrillators"}

Studien zeigen, dass Implantable cardioverter-defibrillators (ICD) Bradykardien effektiv vorbeugen und potenziell tödliche ventrikuläre Arrhythmien unterbinden können [10, 11]. Die Implantation eines ICD ist laut Guidelines der European Society of Cardiology (ESC) eine Klasse-IA-Indikation zur Sekundärprävention des plötzlichen Herztods bei ventrikulären Arrhythmien mit hämodynamischer Instabilität und zur Primärprävention bei symptomatischen $\mathrm{HI}-\mathrm{Pa}$ tienten trotz einer seit mindestens 3 Monaten bestehenden OMT und bei eine LVEF $\leq 35 \%$ geeignet [9]. 


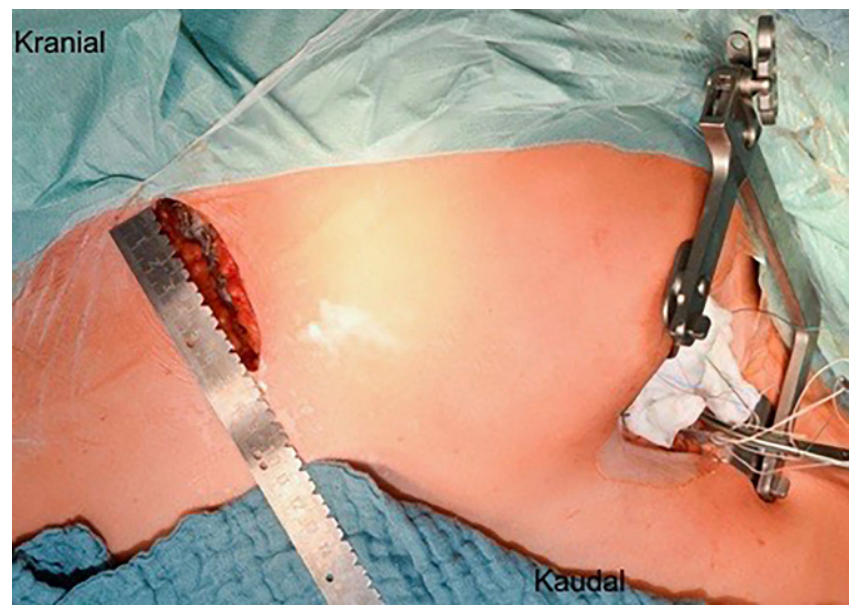

\section{Kardiale Resynchronisations- therapie}

Die CRT mithilfe eines Device kommt für Patienten mit verlängertem QRSKomplex und Linksschenkelblock infrage (Klasse-IA-Indikation). Laut aktueller Studienlage kann bei gut selektierten Patienten die kardiale Leistung verbessert sowie eine Reduktion von Morbidität und Mortalität erreicht werden [12, 13].

\section{Interventionelle Herzinsuffizienz- therapie}

Ähnlich der „transcatheter aortic valve implantation" (TAVI) im Bereich der Aortenklappenvitien besteht auch im Bereich der Herzinsuffizienz die Möglichkeit der interventionellen Therapie, v. a. bei funktioneller Mitralklappeninsuffizienz mit eingeschränkter linksventrikulärer Funktion. In den rezenten ESC Guidelines zur Behandlung von Herzklappenerkrankungen finden sich diese Therapiemöglichkeiten derzeit nur mit einer IIb-Empfehlung, sollte ein chirurgischer Eingriff als zu risikoreich eingestuft werden [14]. Dies ist insbesondere durch die noch als sehr kontrovers einzustufende Studienlage (COAPT vs. MITRA-FR) bedingt. Dennoch ist diese Therapieform als weiteres Tool im Armamentarium des Herzinsuffizienzchirurgen für sorgfältig im Heart-Team ausgewählte Patienten anzusehen.

Derzeit werden folgende 4 Techniken in der interventionellen Therapie der Mitralklappeninsuffizienz angewendet:

- "edge-to-edge repair" (MitraClip" Abbott, Chicago, IL, USA; PASCAL,
Abb. $1<\mathrm{Mi}-$ nimalinvasive Implantation eines „left ventricular assist device" über eine obere Hemisternotomie und eine linksseitige Minithorakotomie

Herzunterstützung und

Herztransplantation

\section{Kurzzeitoptionen}

Aufgrund der technischen und medizinischen Innovationen konnte sich das Gebiet der kurzeitigen Herzunterstützung in den letzten Jahren deutlich weiterentwickeln. Patienten profitieren zunehmend von den verminderten Komplikationsraten und der gesteigerten medizinischen Erfahrung.

Derzeit existieren verschiedene Optionen auf dem Markt der temporären Herzunterstützung. Die älteste, aber nach wie vor sehr bewährte Methode ist die extrakorporale Membranoxygenierung (ECMO), die rein zur respiratorischen Unterstützung (venovenös), aber auch zur kardiorespiratorischen Unterstützung (venoarteriell) herangezogen werden kann. Bei der ECMO handelt es sich um eine effektive Therapie des kardiogenen Schocks (,acute“ oder „acuteon-chronic heart failure"); die ECMOImplantation kann sehr schnell erfolgen und bedarf nicht einmal eines OP oder einer Narkose. Die Nachteile liegen v.a. in der relativ hohen Komplikationsrate (Thromboembolien, Blutungen, Infektionen und Gefäßverletzungen), die mit Dauer der Unterstützung stark ansteigt. Weiterhin können die Patienten unter ECMO-Therapie nur schwer mobilisiert werden (Leistenkanülierung). Sogenannte Wach-ECMO und alternative Kanülierungsstrategien über die obere Körperhälfte sind vierversprechende Verfahren, die in erfahrenen Zentren bereits regelmäßig angewendet werden [17].

er funktioneller Mitralklappenin fizienz ist jedoch fragwürdig, zumindest in Bezug auf den Endpunkt des „left ventricular remodeling " $[15,16]$. Primär sollte der Erhalt der Herzfunktion im Vordergrund der jeweiligen Therapie stehen. Setzt jedoch das Endorganversagen ein, ist die Implantation von Herzunterstützungssystemen oder die Herztransplantation die geeignete Therapieform für die terminale Herzinsuffizienz.

\section{》) Die älteste, aber nach wie vor sehr bewährte Methode ist die extrakorporale Membranoxygenierung}

Ein weiteres Problem liegt in der Tatsache, dass der oftmals schwer geschädigte linke Ventrikel unter der ECMO-Therapie im kardiogenen Schock nicht ausreichend entlastet werden kann und somit die Gefahr einer weiteranhaltenden pulmonalvenösen Stauung besteht bzw. das 
Risiko für eine Stase und Thrombosebildung innerhalb des linken Ventrikels ansteigt. Dieser Problematik kann mithilfe temporärer Herzunterstützungspumpen entgegengewirkt werden, die speziell für den linken Ventrikel konzipiert wurden, wie z. B. die Impella-Pumpe (Fa. Abiomed, Danvers, MA, USA). Diese Pumpen sind in verschiedenen Größen erhältlich, wobei solche mit höheren Flussraten (ca. 51/min) ausschließlich chirurgisch implantiert werden können. Ebenfalls möglich ist der kombinierte Einsatz von ECMO und Impella.

Ziel der Implantation aller temporären Herzunterstützungssysteme ist es, $\mathrm{Pa}$ tienten in einer akut lebensbedrohlichen Herzinsuffizienz zu stabilisieren und daraus resultierende Endorganschäden zu minimieren bzw. zu reversieren. Dies verschafft den behandelnden Ärzten zusätzlich Zeit, um über nachfolgende Therapiestrategien $\mathrm{zu}$ entscheiden (Weaning, "bridge to recovery“" „bridge to bridge“, „bridge to transplantation“, „, bridge to decision").

\section{Langzeittherapie}

Mechanische Pumpen als Herzunterstützungssysteme („ventricular assist device“ [VAD], „total artifical heart“, TAH) haben in den letzten Jahren zur Therapie der terminalen Herzinsuffizienz deutlich an Bedeutung gewonnen. Mittlerweile werden mit steigender Tendenz ca. 1200 Implantationen/Jahr in Deutschland durchgeführt. Der größte Vorteil dieser Systeme gegenüber einer Herztransplantation ist ihre permanente Verfügbarkeit, die die sofortige Implantation auch in dringenden Fällen ermöglicht und ebenfalls $\mathrm{Pa}$ tientengruppen zur Verfügung steht, die nicht die Transplantationskriterien erfüllen.

\section{》) Die Transplantation bleibt der Goldstandard der Langzeittherapie der terminalen Herzinsuffizienz}

Die technologische, medizinische und chirurgische Entwicklung der Herzunterstützungssysteme hat in den letzten Jahren zu einer erhöhten Therapiesicher-

Z Herz- Thorax- Gefäßchir 2020 · 34:62-68 https://doi.org/10.1007/s00398-019-00350-5

(c) Der/die Autor(en) 2019

J. Riebandt · J. S. Hanke

Der Herzinsuffizienzchirurg - Hausarzt und Herzchirurg

Zusammenfassung

Bei der Therapie der terminalen Herzinsuffizienz handelt es sich um einen Wachstumsmarkt - sowohl für Kardiologen als auch für Herzchirurgen. Der besondere Reiz der Arbeit eines Herzinsuffizienzchirurgen besteht in der notwendigen Multidisziplinarität und der Tatsache, dass es nicht nur um den rein mechanistischen Zugang im Sinne der chirurgischen Therapie geht. Vielmehr macht ein Großteil der Arbeit die Vor- und Nachbehandlung aus, die meist durch den Chirurgen selbst ausgeführt wird. Umfassende herzchirurgische Kenntnisse sind ebenso unumgänglich wie das Verständnis kardiologischer und intensivmedizinischer Therapiekonzepte, um dem Patienten eine individuelle und sinnvolle Therapie anbieten zu können. Der vorliegende Beitrag bietet interessierten Kollegen einen Überblick über die Möglichkeiten und Anforderungen in diesem Teilgebiet der Herzchirurgie.

\section{Schlüsselwörter}

Myokardiale Revaskularisation · Herzunterstützungssysteme · Herztransplantation . Behandlerrolle · Klinische Kompetenz

\section{The heart failure surgeon—general practitioner and cardiac surgeon}

\begin{abstract}
The treatment of terminal heart failure is a growing market not only for cardiologists but also for cardiac surgeons. The difference to conventional cardiac surgery is the absolute need for true multidisciplinarity and the fact that the actual surgical part is only a small piece in the puzzle of the management of these patients. Adequate preoperative and postoperative ambulatory treatment is the key to success and is often provided by the cardiac surgeon. As a heart failure surgeon not only comprehensive cardiac surgical skills are required but also a deep understanding
\end{abstract}

of cardiological and intensive care treatment concepts in order to be able to provide the optimal treatment to each specific patient. This article provides an overview of the possibilities and requirements for colleagues interested in a career as a heart failure surgeon.

\section{Keywords}

Myocardial revascularization · Heart-assist devices · Heart transplantation · Professional role Clinical competence heit geführt. Herzunterstützungssysteme sind mittlerweile weniger invasiv implantierbar und über 10-jährige Laufzeiten sind möglich (• Abb. 1). Die Raten der Komplikationen, wie Pumpenthrombosen, gastrointestinale Blutungen und Infektionen, konnten in den letzten Jahren gesenkt werden. Daher entscheiden sich Kardiologen und Chirurgen immer häufiger zum früheren Beginn der VADTherapie, was durch die verbesserte Patientenselektion ebenfalls zu verbesserten Therapieergebnissen führt. Dennoch ist der Einsatz von Herzunterstützungssystemen weiterhin stark von der Patientenselektion und der Compliance der Patienten abhängig. Gleichfalls können Infektionen und thrombembolische
Komplikationen den Therapieverlauf negativ beeinflussen.

Transplantatversagen, biventrikuläres Versagen oder restriktive Kardiomyopathie sind die häufigsten Indikationen zur TAH-Implantation. Das heutzutage einzige aktuell zugelassene TAH ist das pneumatisch betriebene SynCardia TAH $^{\circledR}$ (Fa. Syncardia Systems, U.S.A.). Alternativen hierzu sind die „Off-label“Anwendung von 2 VAD als „biventricular assist device" (BiVAD; [18, 19]) oder 2 VAD nach Kardiektomie in TAHKonfiguration [20]. Diese Operationsmethoden sind jedoch aufgrund der Seltenheit der Erkrankungen, der maximalen Invasivität der Operation und der hohen Komplikationsrate wenig verbreitet. 
Zwar erzielen Herzunterstützungssysteme aufgrund der zahlreichen technischen, medizinischen und chirurgischen Entwicklungen in den vergangenen Jahren Zweijahresüberlebensraten, die denen der Herztransplantation (HTX) vergleichbar sind [21], jedoch bleibt die Transplantation aktuell der Goldstandard der Langzeittherapie der terminalen Herzinsuffizienz. Obwohl es bis dato keine randomisierten Studien zum Thema gibt, ist klar, dass bei richtiger Patientenselektion - ein signifikanter Benefit bezüglich Langezeitüberleben, funktioneller Kapazität und Lebensqualität durch eine HTX zu erwarten ist [9]. Der größte Nachteil der HTX bleibt jedoch ihre limitierte Verfügbarkeit aufgrund des Spenderorganmangels, verglichen mit der Zahl der wartenden Patienten. Laut dem letzten Jahresbericht von Eurotransplant (ET) war die Zahl der aktiv gelisteten Patienten innerhalb des gesamten ETRaums fast doppelt so hoch wie die Zahl der durchgeführten Transplantationen. Es gibt innerhalb der ET-Gemeinschaft jedoch signifikante regionale Unterschiede, z.T. durch die unterschiedliche Gesetzeslage der jeweiligen Mitgliedsstaaten bedingt (Widerspruchsregelung vs. Zustimmungserklärung). Auch ist nicht jeder Patient für eine HTX geeignet. Kontraindikationen wie ein hohes Alter (relative Kontraindikation), pulmonalarterielle Hypertension, Tumorerkrankungen (<5 Jahre), Übergewicht oder Tabakkonsum verbieten eine HTX. Dem weltweit weiter herrschenden Organspendermangel steht allerdings die permanente Verfügbarkeit der Herzunterstützungssysteme gegenüber. Die oben genannte Patientengruppen sowie Patienten, für die die Wartezeit auf eine HTX aufgrund eines akuten Geschehens zu lang ist, können daher für eine VAD-/ Kunstherz-Implantation evaluiert werden. Langzeitkomplikationen der HTX, wie z. B. Hypertonie, Malignome, Nierenschädigungen oder Graft-Vaskulopathie, sind v.a. aufgrund der lebenslangen Einnahme der Immunsuppression $\mathrm{zu}$ erwarten.

Sowohl für den Therapieerfolg bei mechanischer Herzunterstützung als auch bei der HTX ist die kontinuierliche Lang- zeitbetreuung der Herzinsuffizienzpatienten von größter Bedeutung. Diese findet in der an die Klinik angebundenen Herzinsuffizienzambulanz statt. Für die Patienten sind die Ambulanzärzte und Herzchirurgen die Hauptansprechpartner bezüglich der postoperativen Therapie bis hin zu ihrem Versterben. In der Ambulanz erfolgen regelmäßige echokardiographische Untersuchungen, Leistungstests, Biopsien und Anpassungen der Medikation, die der Kardiologe als auch durch der Herzinsuffizienzchirurg durchführt. In den Ambulanzen werden Immunsuppressivaspiegel eingestellt und Kunstherzparameter angepasst. Der Herzinsuffizienzchirurg ist primärer Ansprechpartner bei jeglichen Komplikationen der jeweiligen Therapieform und organisiert deren weiterführende Behandlung. Es erfolgen die regelmäßige Besprechung des Therapieziels und ggf. die Anpassung der Therapie. So wird z. B. mit Kunstherzpatienten und deren Familien die evtl. Listung für eine Herztransplantation besprochen, ebenso wie die evtl. Begrenzung der Therapie bei Komplikationen.

\section{Berufsbild und erforderliche Skills}

\section{Betätigungsgebiet}

Grundsätzlich umfasst die Bezeichnung Herzinsuffizienzchirurgie ein sehr umfangreiches und breit gefächertes Betätigungsgebiet und schließt sowohl die chirurgischen Therapie als auch die ambulante Vor- und Nachbehandlung der Patienten gleichwertig ein.

Immer relevanter wird die Therapie der terminalen Herzinsuffizienz, ebenso wie die Behandlung jener Patienten, die trotz OMT und stetiger Weiterentwicklung der medikamentösen Optionen symptomatisch bleiben. Hier kommen die oben bereits erwähnten Therapiekonzepte der mechanischen Herzunterstützung und des Herzersatzes (mechanisch oder Transplantation) in Betracht. Um die Indikationen für diese Therapieoptionen sinnvoll zu stellen und die Therapien sicher zum Einsatz bringen zu können, sind chirurgische Fähigkeiten und ins- besondere fundiertes theoretisches Hintergrundwissen nötig.

Wie auch in anderen Therapiegebieten der terminalen Herzinsuffizienz ist v.a. der "team approach" von großer Bedeutung. Interdisziplinarität und die enge Zusammenarbeit mit angrenzenden Fachgebieten spielen eine wichtige Rolle im Alltag der Herzinsuffizienzspezialisten. Im besten Fall entscheidet der Herzinsuffizienzchirurg im Team gemeinsam mit Kardiologen, Intensivmedizinern und "heart failure nurses“ über die Weiterbehandlung der Patienten. Anhand klar definierter Leitlinien werden die möglichen Therapieformen diskutiert. Insbesondere ein guter ArztPatient-Kontakt prägt die Therapie maßgeblich, da diese an den individuellen Patienten angepasst werden muss.

\section{》) Wegen des komplexen und anspruchsvollen Patienten- kollektivs ist Teamarbeit der Schlüssel zum Erfolg}

Auch nach der Operation ist Teamarbeit der Schlüssel zum Erfolg, da wohl kaum ein komplexeres und anspruchsvolleres Patientenkollektiv existiert. Damit der Erfolg der Operation möglichst lange erhalten bleibt, bedarf es der engen Zusammenarbeit zwischen Herzchirurgen, Anästhesisten und Intensivmedizinern sowie Kardiologen, aber auch VAD-Koordinatoren/Kardiotechnikern und Heart failure nurses bzw. dem Pflegepersonal der Stationen und der Ambulanzen. Um dem komplexen Patientengut gerecht $\mathrm{zu}$ werden und eine umfassende Betreuung zu gewährleisten, werden immer häufiger „heart failure units“ gebildet, in denen alle zuvor genannten Spezialisten gebündelt aufeinandertreffen und nicht nur theoretisch, sondern auch räumlich eine Einheit bilden.

\section{Skills-Set}

\section{Literaturkenntnis}

Ein umfassender Überblick über die aktuellen Literatur- und Forschungsergebnisse ist für den Herzinsuffizienzchirurgen unumgänglich, um eine geeignete 
Patientenselektion und Indikationsstellungen bezüglich der Device-Auswahl sicherzustellen und ggf. die richtige Entscheidung zwischen mechanischer Herzunterstützung und Herztransplantation zu treffen. Derzeit relevant sind diverse Studien zu aktuellen LVAD (ROADMAP, MOMENTUM, ELEVATE, ReVOLVE, ENDURANCE Supplemental; [22-26]) und zur MitraClip-Therapie (COAPT, MITRA-FR; $[27,28]$ ). Aber auch ältere Landmarkpublikationen sollten bekannt sein $[29,30]$.

Neue Studienergebnisse werden oft auf einschlägigen Fachkonferenzen und Symposien diverser Fachgesellschaften präsentiert. Im Folgenden soll eine kleine Auswahl relevanter europäischer und amerikanischer Gesellschaften bzw. Treffen genannt werden, die zumeist in eigenen themenspezifischen Journale publizieren: Jahrestagung der Deutschen Gesellschaft für Thorax-, Herzund Gefäßchirurgie, Jahrestagung der International Society of Heart and Lung Transplantation (ISHLT), Jahrestagung der European Association for CardioThoracic Surgery (EACTS) und European Mechanical Circulatory Support Summit (EUMS).

\section{Theoretisches und fach- übergreifendes Wissen}

Ein Blick über den Tellerrand empfiehlt sich immer, allein schon, um in allfälligen Diskussionen sachlich fundiert über das beste Vorgehen bei einem Patienten argumentieren zu können. Im Bereich der Herzinsuffizienz sind v. a. Kenntnisse in der kardiovaskulären Pathophysiologie relevant, und ein tiefes Verständnis darüber, wo kardiologische Therapiekonzepte jeweils einzugreifen versuchen. Von immenser Wichtigkeit ist außerdem fundiertes Wissen über intensivmedizinische Maßnahmen und deren Auswirkungen, wie z.B. die korrekte Anwendung vasoaktiver und inotroper Medikamente, invasiver und nichtinvasiver Beatmungskonzepte oder der Einsatz von Organersatztherapien. Da die Ausbildungsordnung der Herzchirurgie z. B. in Österreich im Gegensatz zu Deutschland derzeit keine Ausbildungszeit auf der Anästhesie oder Intensivstation vorsieht, ist ein gewisses Eigenengagement gefordert.

\section{Klinische Skills}

Analog zur benötigten fachlichen Vielfalt auf dem Gebiet der Herzinsuffizient sollten auch die klinischen Skills eines Herzinsuffizienzchirurgen breit aufgestellt sein. Diese reichen von Echokardiographiekenntnissen über „wire skills“ (z. B. bei ECMO- oder Impella-Implantation oder das Durchführen einer Myokardbiopsie) bis hin zur Anwendung minimalinvasiver Operationstechniken bei der Implantation von herzunterstützenden Pumpen. Eine fundierte allgemein herzchirurgische Ausbildung ist selbstverständliche Voraussetzung.

Neben den operativen Fähigkeiten sind die Kenntnis und korrekte Anwendung medikamentöser Therapien bei der Betreuung von LVAD- oder Transplantationspatienten förderlich und wichtig für den Therapieerfolg. So kann z. B. die Qualität der Blutdrucktherapie und der eingestellten Antikoagulation zwischen einem guten Langzeitverlauf oder einer schweren neurologischen Komplikation bei Schlaganfall oder Hirnblutung entscheiden, oder falsch-dosierte Immunsuppressiva können eine schwere Abstoßung oder massive Infektion auslösen.

\section{》) Die Arbeit des Herzinsuf- fizienzchirurgen schließt das mehrjährige postoperative Patientenmanagement ein}

Einen Großteil der Nachbetreuung - stationär oder ambulant - macht die Prävention oder auch die Behandlung schwerwiegender Therapiekomplikationen aus. Die Arbeit des Herzinsuffizienzchirurgen endet also nicht bei Nahtende im OP, sondern beinhaltet im Gegensatz zur traditionellen Herzchirurgie auch und insbesondere das mehrjährige postoperative Management der Patienten bis hin $\mathrm{zu}$ Therapiebegrenzung und Sterbebegleitung.

\section{Industrie und Forschung}

Die Produkthersteller sind wichtige Partner des Herzinsuffizienzchirurgen. Die Vielzahl von technischen Geräten erfordert die gute Zusammenarbeit zwischen "clinical specialists“, Ingenieuren und Handelspartnern und Chirurgen. Der große Umfang an neuen Technologien macht zahlreiche Schulungen und Einarbeitungen in die neuen Geräte notwendig.

Eine wichtige Rolle kommt Industriepartnern im Bereich "troubleshooting“ zu. Die Kenntnis der Logfiles der jeweiligen Geräte und die Rücksprache mit der jeweiligen Firma bzw. Technikern liefern oft hilfreiche Informationen für die Lösung klinisch relevanter Probleme. Auch in Bezug auf Fortbildungsmöglichkeiten bieten die jeweiligen Firmen mehrfach jährlich verschiedene Implantationskurse und "user meetings" an. Diese richten sich meist nicht nur an Chirurgen, sondern sind interdisziplinär, was wiederum den Alltag der Herzinsuffizienztherapie gut widerspiegelt.

Die kontinuierliche Weiterentwicklung im Bereich der Herzinsuffizienztherapie hat das Spektrum der Forschungsmöglichkeiten deutlich erweitert. Grundlagenforschung im Bereich der Immunsuppression und Medikation ist ebenso möglich wie die Entwicklung von Software, neuen chirurgischen Techniken und neuen Technologien. Neben klinischen Studien im kardiologischen und im kardiochirurgischen Bereich hat die zunehmende Zahl von Herzinsuffizienzpatienten dazu geführt, dass viele andere medizinische Fachbereiche nun Forschung in diesem Bereich betreiben. So bietet die chirurgische Therapie der Herzinsuffizienz ein breites Spektrum an Möglichkeiten, um sich wissenschaftlich mit einem hochinnovativen Themengebiet zu beschäftigen.

\section{Fazit und Perspektive}

- Die Herzinsuffizienz stellt einen Wachstumsmarkt dar. Aufgrund des Fortschritts von medizinischer Forschung, chirurgischen Techniken und technologischer Innovation hat sich in den letzten Jahren das Behandlungsspektrum der Herzinsuffizienz deutlich erweitert.

- Die Herzinsuffizienzchirurgie bildet einen zukunftsträchtigen und innovativen Bereich der Herzchirurgie. Die allumfängliche Betreuung der $\mathrm{Pa}$ - 


\section{tienten von Diagnose über Therapie bis zur Führsorge bis hin zum Lebens- ende des Patienten unterscheidet die Herzinsuffizienzchirurgie von der klassischen Herzchirurgie. \\ - Die enge Zusammenarbeit im Heart- Team mit Kardiologen, „heart failure nurses", Intensivmediziner und Indus- triepartnern ermöglicht den Aufbau von Teamstrukturen zur Behandlung der Herzinsuffizienz und macht den Beruf des Herzinsuffizienzchirurgen vielseitig und attraktiv.}

\section{Korrespondenzadresse}

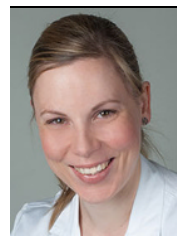

\section{Dr. Julia Riebandt}

Klinische Abteilung für Herzchirurgie, Universitätsklinik für Chirurgie, Medizinische Universität Wien Währinger Gürtel 18-20, 1090 Wien, Österreich Julia.riebandt@ meduniwien.ac.at

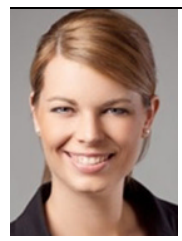

\section{Dr. Jasmin S. Hanke}

Abteilung für Herz-, Thorax-, Transplantations- und Gefäßchirurgie, Medizinische Hochschule Hannover Carl-Neuberg-Str. 1, 30625 Hannover,

Deutschland Hanke.Jasmin@ mh-hannover.de

Dr. Julia Riebandt hat in Wien Humanmedizin studiert. Seit 2012 arbeitet sie als wissenschaftliche Mitarbeiterin im VAD-Programm der Medizinischen Universität Wien und ist seit 2013 ärztlich an der Abteilung für Herzchirurgie der Medizinischen Universität Wien tätig. Sie hat sowohl eine klinische als auch wissenschaftliche Laufbahn im Bereich der Herzchirurgie mit Fokus auf terminale Herzinsuffizienz, mechanische Herzunterstützung und Herztransplantation eingeschlagen.

Dr. Jasmin S. Hanke hat in Düsseldorf Humanmedizin studiert. Seit 2012 arbeitet sie in der Abteilung für Herz-, Thorax-, Transplantations- und Gefäßchirurgie in der Medizinischen Hochschule Hannover. Sie hat sowohl eine klinische als auch wissenschaftliche Laufbahn im Bereich der Herzchirurgie mit Fokus auf terminale Herzinsuffizienz, mechanische Herzunterstützung und Herztransplantation eingeschlagen.

Funding. Open access funding provided by Medical University of Vienna.
Einhaltung ethischer Richtlinien

Interessenkonflikt. J. Riebandt und J.S. Hanke geben an, dass kein Interessenkonflikt besteht.

Für diesen Beitrag wurden von den Autoren keine Studien an Menschen oder Tieren durchgeführt. Für die aufgeführten Studien gelten die jeweils dort angegebenen ethischen Richtlinien.

Open Access. Dieser Artikel wird unter der Creative Commons Namensnennung 4.0 International Lizenz veröffentlicht, welche die Nutzung, Vervielfältigung, Bearbeitung, Verbreitung und Wiedergabe in jeglichem Medium und Format erlaubt, sofern Sie den/die ursprünglichen Autor(en) und die Quelle ordnungsgemäß nennen, einen Link zur Creative Commons Lizenz beifügen und angeben, ob Änderungen vorgenommen wurden.

Die in diesem Artikel enthaltenen Bilder und sonstiges Drittmaterial unterliegen ebenfalls der genannten Creative Commons Lizenz, sofern sich aus der Abbildungslegende nichts anderes ergibt. Sofern das betreffende Material nicht unter der genannten Creative Commons Lizenz steht und die betreffende Handlung nicht nach gesetzlichen Vorschriften erlaubt ist, ist für die oben aufgeführten Weiterverwendungen des Materials die Einwilligung des jeweiligen Rechteinhabers einzuholen.

Weitere Details zur Lizenz entnehmen Sie bitte der Lizenzinformation auf http://creativecommons.org/ licenses/by/4.0/deed.de.

\section{Literatur}

1. destatis (Hrsg) (2019) Die 10 häufigsten Todesfälle durch Herz-Kreislauf-Erkrankungen. https://www.destatis.de/DE/ZahlenFakten/ GesellschaftStaat/Gesundheit/Todesursachen/ Tabellen/HerzKreislaufErkrankungen.html. Zugegriffen:26. Sept. 2019

2. Körfer R, ArusogluL (2010) Herzunterstützungssysteme und Kunstherzimplantation. Herzchirurgie. Springer, Berlin, Heidelberg

3. Deutsche Herzstiftung e. V. (2017) Jahresbericht 2017

4. Filippatos G, Khan SS, Ambrosy AP et al (2015) International REgistry to assess medical Practice with IOngitudinal obseRvation for Treatment of HeartFailure (REPORT-HF): rationale for and design of a global registry. Eur J Heart Fail 17:527-533

5. Tiller D, Russ M, Greiser KH et al (2013) Prevalence of symptomatic heart failure with reduced and with normal ejection fraction in an elderly general population-the CARLA study. PLoSONE 8:e59225

6. Redfield MM, Jacobsen SJ, Burnett JC Jr., Mahoney DW, Bailey KR, Rodeheffer RJ (2003) Burden of systolic and diastolic ventricular dysfunction in the community: appreciating the scope of the heart failure epidemic. JAMA 289:194-202

7. Maggioni AP, Dahlstrom U, Filippatos G et al (2013) EURObservational Research Programme: regional differences and 1-year follow-up results of the Heart Failure Pilot Survey (ESC-HF Pilot). Eur J Heart Fail 15:808-817

8. Crespo-Leiro MG, Anker SD, Maggioni AP et al (2016) European Society of Cardiology Heart Failure Long-Term Registry (ESC-HF-LT): 1-year follow-up outcomes and differences across regions. Eur J Heart Fail 18:613-625

9. Ponikowski P, Voors AA, Anker SD et al (2016) 2016 ESC Guidelines for the diagnosis and treatment of acute and chronic heart failure: The Task Force for the diagnosis and treatment of acute and chronic heart failure of the European Society of Cardiology (ESC). Developed with the special contribution of the Heart Failure Association (HFA) of the ESC. Eur J HeartFail 18:891-975

10. Kuck KH, Cappato R, Siebels J, Ruppel R (2000) Randomized comparison of antiarrhythmic drug therapy with implantable defibrillators in patients resuscitated from cardiac arrest: the Cardiac Arrest Study Hamburg (CASH). Circulation 102:748-754

11. Kadish A, Dyer A, Daubert JP et al (2004) Prophylactic defibrillator implantation in patients with nonischemic dilated cardiomyopathy. N Engl JMed 350:2151-2158

12. Sohaib SM, Finegold JA, Nijjer SS et al (2015) Opportunity to increase life span in narrow QRS cardiac resynchronization therapy recipients by deactivating ventricular pacing: evidence from randomized controlled trials. JACC Heart Fail 3:327-336

13. Cleland JG, Abraham WT, Linde C et al (2013) An individual patient meta-analysis of five randomized trials assessing the effects of cardiac resynchronization therapy on morbidity and mortality in patients with symptomatic heart failure. Eur Heart J 34:3547-3556

14. Baumgartner H, FalkV, Bax JJ et al (2017) 2017 ESC/ EACTS Guidelines for the management of valvular heart disease. Eur Heart J 38:2739-2791

15. Michler RE, Smith PK, Parides MK et al (2016) Twoyear outcomes of surgical treatment of moderate Ischemic mitral regurgitation. $\mathrm{N}$ Engl J Med 374:1932-1941

16. Goldstein D, Moskowitz AJ, Gelijns AC et al (2016) Two-year outcomes of surgical treatment of severe Ischemic mitral regurgitation. $\mathrm{N}$ Engl J Med 374:344-353

17. Abrams D, Garan AR, Brodie D (2019) Awake and fully mobile patients on cardiac extracorporeal life support. Ann Cardiothorac Surg 8:44-53

18. Lavee J, Mulzer J, Krabatsch T et al (2018) An international multicenter experience of biventricular support with HeartMate 3 ventricular assist systems. JHeart Lung Transplant 37:1399-1402

19. Strueber M, Meyer AL, Malehsa D, Haverich $A$ (2010) Successful use of the HeartWare HVAD rotary blood pump for biventricular support. JThorac Cardiovasc Surg 140:936-937

20. Daneshmand MA, Bishawi M, Milano CA, Schroder JN (2019) The HeartMate 6. ASAIO J https://doi. org/10.1097/MAT.0000000000001011

21. Mehra MR, Uriel N, Naka Y et al (2019) A fully magnetically levitated left ventricular assist device-final report. NEngl J Med 380:1618-1627

22. Shah KB, Starling RC, Rogers JG et al (2018) Left ventricular assist devices versus medical management in ambulatory heart failure patients: An analysis of INTERMACS Profiles 4 and 5 to 7 from the ROADMAP study. J Heart Lung Transplant 37:706-714

23. Mehra MR, Goldstein DJ, Uriel N et al (2018) Two-year outcomes with a magnetically levitated cardiac pump in heart failure. N Engl J Med 378:1386-1395

24. Garbade J, Gustafsson F, Shaw S et al (2019) Postmarket experience with heartmate 3 left ventricular assist device: 30-day outcomes from the ELEVATE registry. Ann Thorac Surg 107:33-39 
25. Schmitto JD, Zimpfer D, Fiane AE et al (2016) Longterm support of patients receiving a left ventricular assist device for advanced heart failure: a follow-up analysis of the Registry to Evaluate the HeartWare Left Ventricular Assist System. Eur J Cardiothorac Surg 50:834-838

26. Milano CA, Rogers JG, Tatooles AJ et al (2018) HVAD: the ENDURANCE supplemental trial. JACC Heart Fail 6:792-802

27. Stone GW, Lindenfeld J, Abraham WT et al (2018) Transcatheter mitral-valve repair in patients with heart failure. NEngl J Med 379:2307-2318

28. Obadia JF, Messika-Zeitoun D, Leurent $G$ et al (2018) Percutaneous repair or medical treatment for secondary mitral regurgitation. N Engl J Med 379:2297-2306

29. Slaughter MS, Rogers JG, Milano CA et al (2009) Advanced heart failure treated with continuousflow left ventricular assist device. N Engl J Med 361:2241-2251

30. RoseEA, Gelijns AC, MoskowitzAJ etal (2001) Long term use of a left ventricular assist device for endstage heart failure. N Engl J Med 345:1435-1443

\section{Künstliche Intelligenz auf Augenhöhe}

Metaanalyse zur medizinischen Bildgebung

Künstliche Intelligenz contra Mediziner: Beide erreichen inzwischen ähnliche Trefferquoten beim Erkennen von Krankheiten.

Hohe Trefferquote: Künstliche Intelligenz (KI) hat $87 \%$ der Krankheiten richtig erkannt bei den Ärzten waren es im Vergleich dazu 86\%. Umgekehrt habe KI in 93\% der Fälle richtigerweise eine Krankheit des Patienten ausgeschlossen - hier seien $91 \%$ der Ärzte zum selben Ergebnis gekommen. Bei der Diagnostik von Krankheiten begegnet $\mathrm{KI}$ somit dem leibhaftigen Arzt zumindest auf Augenhöhe, so das Ergebnis der ersten systematischen Metaanalyse von 14 Studien.

\section{Wenig qualitativ hochwertige Studien}

Wie Studienleiter Prof. Alastair Denniston vom University Hospital Birmingham erläutert, habe sein Team um den Erstautoren Liu Xiaoxuan mehr als 20.500 wissenschaftliche Beiträge zur KI-basierten Diagnostikleistung innerhalb der Jahre 2012 bis 2019 identifiziert, wovon allerdings weniger als $1 \%$ für aussagekräftig befunden worden seien. Nur die anfangs erwähnten 14 Studien verglichen die Resultate der KI mit denen der Ärzte.

Innerhalb dieser Handvoll qualitativ hochwertiger Studien habe sich gezeigt, dass mittels Deep Learning in der Tat Krankheiten in der Bandbreite von Augenerkrankungen bis hin zu Krebs erkannt werden konnten und zwar genauso zuverlässig wie von Ärztehand, verdeutlicht Denniston. Aber es müsse auch erwähnt werden, dass KI die menschliche Diagnostik nicht wesentlich outperformt hat, ergänzt er. Deep Learning ist dabei eine Form des maschinellen Lernens, bei dem die Funktionsweise dem menschlichen Gehirn nachempfunden wird.

\section{Hightech-Strategie 2025 setzt auf KI}

Die Erkenntnis ist nicht unwichtig: Denn die EU-Kommission investiert zum Beispiel 35 Millionen Euro in die Prävention, Vorhersage und Behandlung der häufigsten Krebsarten mithilfe der Kl. Auch die Bundesregierung setzt in ihrer Hightech-Strategie 2025 explizit auf Kl, um Krebs zu bekämpfen.
Generell fordern die Autoren eine bessere Studienqualität ein, um die Leistungsfähigkeit der KI im Vergleich zur ärztlichen Diagnosefähigkeit fundierter bewerten zu können. Allen voran fehle es an entsprechenden Rahmenvorgaben für wissenschaftliches Arbeiten. Allerdings sei bereits eine Qualitätssteigerung bei jüngeren Studien zu beobachten.

Quelle: Ärzte Zeitung basierend auf: The Lancet Digital Health (2019) 1(6):271-297 